\title{
TRANSFORMATIONS OF FOURIER COEFFICIENTS
}

\author{
FREDERICK H. YOUNG ${ }^{1}$
}

Introduction. In $1923 \mathrm{M}$. Fekete [2] ${ }^{2}$ introduced the concept of factor sequences that left invariant the class of a Fourier series. That is, Fekete investigated the conditions to which a sequence of constants $\left(\lambda_{n}\right)$ must be subjected in order that $\left(\lambda_{n} a_{n}, \lambda_{n} b_{n}\right)$ be Fourier coefficients of a function of the same class, $K$, as that of the function determined by $\left(a_{n}, b_{n}\right)$. Whenever $\left(\lambda_{n}\right)$ has this property, $\left(\lambda_{n}\right)$ is said to belong to the class $(K, K)$. Fekete restricted his investigation to those cases for which $K$ represented the class of continuous, essentially bounded, Riemann integrable, or Lebesgue integrable functions, functions of bounded variation, or functions having a LebesgueStieltjes series. It may be mentioned in passing that Verblunsky [5] extended Fekete's results to some of the cross classes.

Note that the transformation effected by a factor sequence may be accomplished by matric multiplication. Suppose that the cosine coefficients of $f$ are $\left(a_{n}\right)$. Then the coefficients of the function resulting from the transformation appear in the column vector on the right below.

$$
\left[\begin{array}{cccc}
\lambda_{0} & 0 & 0 & \cdots \\
0 & \lambda_{1} & 0 & \cdots \\
0 & 0 & \lambda_{2} & \cdots \\
\cdot & \cdots & \cdots & \cdot \\
\cdots & \cdots & \cdots
\end{array}\right] \cdot\left[\begin{array}{c}
a_{0} \\
a_{1} \\
a_{2} \\
\cdot \\
\cdot
\end{array}\right]=\left[\begin{array}{c}
\lambda_{0} a_{0} \\
\lambda_{1} a_{1} \\
\lambda_{2} a_{2} \\
\cdot \\
\cdot
\end{array}\right] .
$$

The purpose of this paper is to extend the diagonal matrix on the left to a full $\infty \times \infty$ matrix with constant elements. We shall investigate such matrices, denoted by $T$, and endeavor to find necessary and sufficient conditions for $T$ to transform the coefficients of a function in the class $L_{p}$ into coefficients of another function in the same class.

G. H. Hardy [3] has shown that the arithmetic means of the partial sums of coefficients of a function in $L_{p}, p \geqq 1$, are also in the same class. Since this transformation can be accomplished by the matrix

Presented to the Society, November 25, 1950; received by the editors August 5, 1951.

${ }^{1}$ AEC Research Fellow, University of Oregon.

2 Numbers in brackets refer to the bibliography at the end of the paper. 


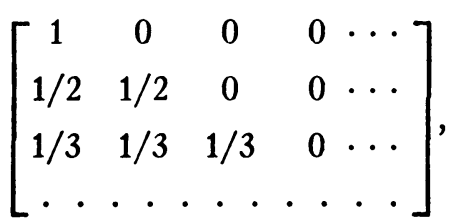

Hardy's paper gives a preliminary result along the present line of investigation. In 1944, Bellman [1] showed that the transpose of the matrix above is also of the same type for $p>1$. In view of Hardy's theorem, Bellman's result is an immediate consequence of Theorem 5 of this paper.

The following treatment will be seen to be complete for only the open interval, $1<p<\infty$. The end points will need a more searching analysis.

Definitions and notation. The letter $T$ will be used to denote both the matrix $\left(a_{k j}\right)$ and the associated transformation. Further, let

$$
\left(a_{j}, b_{j}\right) \sim f \in L_{p}, \quad 1<p<\infty .
$$

The symbol $\sim$ will be used to indicate the correspondence between an integrable function and its Fourier coefficients. Thus, in (2) and (3) below, the indicated correspondence must be justified. With $p$ as in (1),

(2) for each $k$,

$$
\begin{aligned}
& \left(a_{k j}, 0\right) \sim g_{k} \in L_{p^{\prime}} \quad \text { and } \quad G_{n, k}(x)=\sum_{j=0}^{n}\left(\frac{n+1-j}{n+1}\right) a_{k j} \cos j x, \\
& \left(0, a_{k j}\right) \sim h_{k} \in L_{p^{\prime}} \quad \text { and } \quad H_{n, k}(x)=\sum_{j=0}^{n}\left(\frac{n+1-j}{n+1}\right) a_{k j} \sin j x ;
\end{aligned}
$$

(3) for each $j$,

$$
\begin{array}{ll}
\left(a_{k j}, 0\right) \sim p_{j} \in L_{p} \quad \text { and } \quad P_{n, j}(x)=\sum_{k=0}^{n}\left(\frac{n+1-k}{n+1}\right) a_{k j} \cos k x, \\
\left(0, a_{k j}\right) \sim q_{j} \in L_{p} \quad \text { and } \quad Q_{n, j}(x)=\sum_{k=0}^{n}\left(\frac{n+1-k}{n+1}\right) a_{k j} \sin k x ;
\end{array}
$$

(4) $\underset{\sigma_{n}}{T}(f ; x)=\sum_{k=0}^{n}\left(\frac{n+1-k}{n+1}\right) \sum_{j=0}^{\infty} a_{k j}\left(a_{j} \cos k x+b_{j} \sin k x\right)$;

(5) $U_{n}(x ; t)=\sum_{k=0}^{n}\left(\frac{n+1-k}{n+1}\right)\left[g_{k}(t) \cos k x+h_{k}(t) \sin k x\right]$.

There is no loss of generality in assuming that the first row of $T$ 
consists entirely of zeros. Since we shall also discuss $T^{*}$, the transpose of $T$, we shall also let the first column of $T$ consist of zeros. This enables us to use the simpler expression employed in (2) and (3) and, later, for the Fejér means.

The association of function and series in (2) and (3) is so far purely formal. However, in general,

$$
T(f) \sim \sum_{k=0}^{\infty}\left[\sum_{j=0}^{\infty} a_{k j} a_{j} \cos k x+\sum_{j=0}^{\infty} a_{k j} b_{j} \sin k x\right],
$$

and for these coefficients to exist for every $f$ in some $L_{p}, 1<p<\infty$, it is necessary that each row be in $L_{p^{\prime}}$, which justifies (2). Further, if $f$ is specialized to be either $\cos j x$ or $\sin j x$, it is clear that each column must be in $L_{p}$, which justifies (3). We shall say that $T \in\left(L_{p}\right)$ if (1) implies that $T(f) \in L_{p}$.

LEMMA 1. For $1<p<\infty, \sigma_{n}^{T}(f ; x)=(1 / \pi) \int_{0}^{2 \pi} f(t) L_{n}(x ; t) d t$, where $L_{n}(x ; t) \sim\left(P_{n, j}(x), Q_{n, j}(x)\right)$ for each $n$ and $x$.

Proof. By (1) and (2), $\sigma_{n}^{T}(f ; x)$ exists for each $n$ and all $x, 0 \leqq x \leqq 2 \pi$, $1<p<\infty$, and

$$
\begin{aligned}
\underset{\sigma_{n}}{T}(f ; x) & =\sum_{j=0}^{\infty}\left[a_{j} \sum_{k=0}^{n}\left(\frac{n+1-k}{n+1}\right) a_{k j} \cos k x\right. \\
& \left.+b_{j} \sum_{k=0}^{n}\left(\frac{n+1-k}{n+1}\right) a_{k j} \sin k x\right] \\
& =\sum_{j=0}^{\infty}\left[a_{j} P_{n, j}(x)+b_{j} Q_{n, j}(x)\right] .
\end{aligned}
$$

Thus, for each $n$ and $x$, by Parseval's relation,

$$
\left(P_{n, j}(x), Q_{n, j}(x)\right) \sim L_{n}(x ; t) \in L_{p},
$$

and, moreover, $\sigma_{n}^{T}(f ; x)=(1 / \pi) \int_{0}^{2 \pi} f(t) L_{n}(x ; t) d t$.

Lemma 2. $\left\|L_{n}(x ; t)\right\|_{p^{\prime}} \leqq K(n)$ for all $x$, the norm being taken with respect to $t$.

Proof.

$$
\begin{aligned}
L_{n}(x ; t) \sim \sum_{k=0}^{n}\left(\frac{n+1-k}{n+1}\right)\left[\left(\sum_{j=0}^{\infty} a_{k j} \cos j t\right) \cos k x\right. \\
\left.+\left(\sum_{j=0}^{\infty} a_{k j} \sin j t\right) \sin k x\right]
\end{aligned}
$$


Let $\sigma_{m}\left(L_{n}(x ; t) ; t\right)$ be the $m$ th mean of $L_{n}(x ; t)$ with respect to $t$. Then

$$
\sigma_{m}\left(L_{n}(x ; t) ; t\right)=\sum_{k=0}^{n}\left(\frac{n+1-k}{n+1}\right)\left[G_{m, k}(t) \cos k x+H_{m, k}(t) \sin k x\right] .
$$

By Minkowski's inequality, again with respect to $t$,

$$
\begin{aligned}
\left\|\sigma_{m}\left(L_{n}(x ; t) ; t\right)\right\|_{p^{\prime}} & \leqq \sum_{k=0}^{n}\left(\frac{n+1-k}{n+1}\right)\left[\left\|G_{m, k}\right\|_{p^{\prime}}+\left\|H_{m, k}\right\|_{p^{\prime}}\right] \\
& \leqq \sum_{k=0}^{n}\left(\frac{n+1-k}{n+1}\right)\left[\left\|g_{k}\right\|_{p^{\prime}}+\left\|h_{k}\right\|_{p^{\prime}}\right],
\end{aligned}
$$

which is independent of both $x$ and $m$. Hence the lemma follows.

Define $\left\|L_{n}(x ; t)\right\|_{p^{\prime}}=M_{n}(x)$. Then $M_{n}(x) \in B$ for each $n$.

We now have

$$
L_{n}(x ; t) \sim \sum_{k=0}^{n}\left(\frac{n+1-k}{n+1}\right) \sum_{j=0}^{\infty} a_{k j} \cos (k x-j t) .
$$

Recall that

$$
U_{n}(x ; t)=\sum_{k=0}^{n}\left(\frac{n+1-k}{n+1}\right)\left(g_{k}(t) \cos k x+h_{k}(t) \sin k x\right) .
$$

Then the Fourier series of $U_{n}(x ; t)$ is the same as that of $L_{n}(x ; t)$. That is, for each $x, U_{n}(x ; t)=L_{n}(x ; t)$ for almost all $t$. Thus, we may write

$$
\begin{aligned}
\sigma_{n}^{T}(f ; x) & =\frac{1}{\pi} \int_{0}^{2 \pi} f(t) U_{n}(x ; t) d t \\
& =\frac{1}{\pi} \sum_{k=0}^{n}\left(\frac{n+1-k}{n+1}\right) \int_{0}^{2 \pi} f(t)\left[\left(g_{k}(t) \cos k x+h_{k}(t) \sin k x\right] d t .\right.
\end{aligned}
$$

We note that for each $x, U_{n}(x ; t)$ is in $L_{p^{\prime}}$ as a function of $t$, and for each $t, U_{n}(x ; t)$ is a continuous function of $x$.

Theorem 1. If $T \in\left(L_{p}\right)$, then $T$ is a linear bounded transformation.

Proof. $\left|\sigma_{n}^{T}(f ; x)\right| \leqq(1 / \pi)\|f\|_{p} M_{n}(x)$ where $M_{n}(x) \in B .\left\|T_{n}(f)\right\|_{p}$ $=\left\|\sigma_{n}^{T}(f ; x)\right\|_{p} \leqq(1 / \pi)\|f\|_{p} K(n)$. Then $T_{n}$ is a bounded linear transformation for each $n$ with modulus $\leqq K(n) / \pi$. However, $\left\|T_{n}(f)\right\|_{p}$ is bounded for each $f \in L_{p}$. Hence, the sequence of moduli is bounded by the principle of uniform boundedness. Moreover, $T_{n}(f) \rightarrow T(f)$ in the metric of $L_{p}$. Thus, by the Banach-Steinhaus theorem, $T$ is a bounded linear transformation. 
The result we shall use is that $\left\|T_{n}(f)\right\|_{p} \leqq K\|f\|_{p}$ for all $n$.

It is known that $\left\|\sigma_{n}^{T}(f ; x)\right\|_{p}=\sup _{\|\emptyset\|_{p^{\prime}} \leqq 1}\left|\int_{0}^{2 \pi} g(x) \sigma_{n}^{T}(f ; x) d x\right|$. Fix $n$ and $f$. Then a particular $g$ with $\|g\|_{p^{\prime}} \leqq 1$ is determined such that for that $n, f$, and $g$,

$$
\begin{aligned}
\left\|\sigma_{n}^{T}(f ; x)\right\|_{p} & =\left|\int_{0}^{2 \pi} g(x) \underset{\sigma_{n}}{T}(f ; x) d x\right| \\
& =\frac{1}{\pi}\left|\int_{0}^{2 \pi} g(x) \int_{0}^{2 \pi} f(t) U_{n}(x ; t) d t d x\right| \\
& =\frac{1}{\pi}\left|\int_{0}^{2 \pi} f(t)\left\{\int_{0}^{2 \pi} g(x) U_{n}(x ; t) d x\right\} d t\right| .
\end{aligned}
$$

Theorem 2. A necessary and sufficient condition for $T \in\left(L_{p}\right), 1<p$ $<\infty$, is the existence of $K$ such that $\sup _{\|\left.\boldsymbol{l o}\right|_{p^{\prime}} \leqq 1}\left\|\int_{0}^{2 \pi} g(x) U_{n}(x ; t) d x\right\|_{p^{\prime}}$ $\leqq K$ for all $n$.

Proof. Sufficiency. Let $f \in L_{p}$ and fix $n$. Then let $g$ be the function for which (7) holds. Then, by Hölder's inequality, $\left\|\sigma_{n}^{T}(f ; x)\right\|_{p}$ $\leqq(1 / \pi)\|f\|_{p}\left\|\int_{0}^{2 \pi} g(x) U_{n}(x ; t) d x\right\|_{p^{\prime}} \leqq(K / \pi)\|f\|_{p .}$ Thus, $T \in\left(L_{p}\right)$.

Necessity. Let $T \in\left(L_{p}\right)$. Fix $n$ and $g$ with $\|g\|_{p^{\prime}} \leqq 1$ and let $T_{n}(f)$ $=(1 / \pi) \int_{0}^{2 \pi} f(t)\left\{\int_{0}^{2 \pi} g(x) U_{n}(x ; t) d x\right\} d t$. Then $\left|T_{n}(f)\right| \leqq \| \sigma_{n}^{T}\left(f ; x\left\|_{p} \leqq K\right\| f \|_{p}\right.$ by Theorem 1. Thus, $T_{n}(f)$ is a linear functional over $L_{p}$, and, by Banach, its norm is $\left|T_{n}\right|=\left\|\int_{0}^{2 \pi} g(x) U_{n}(x ; t) d x\right\|_{p}{ }^{\prime} \leqq K$ for each $n$ and $g$.

It is now possible to indicate other conditions that are either necessary or sufficient for $T \in\left(L_{p}\right)$.

THEOREM 3. A sufficient condition for $T \in\left(L_{p}\right)$ is given by either

$$
\|\left(\left\|U_{n}(x ; t)\right\|_{\substack{p^{\prime} \\ t}} \|_{\substack{p \\ x}}=O(1)\right.
$$

$$
\left\|\left(\left\|U_{n}(x ; t)\right\|_{\substack{p \\ x}}\right)\right\|_{\substack{p^{\prime} \\ \text { in }}}=O(1) .
$$

Proof. Part (1) follows immediately from an application of Hölder's inequality to the right side of

$$
\left|\sigma_{n}^{T}(f ; x)\right|=\left|\frac{1}{\pi} \int_{0}^{2 \pi} f(t) U_{n}(x ; t) d t\right| .
$$

Part (2) is a corollary to Theorem 2.

Theorem 4. $T \in\left(L_{p}\right)$ implies $\left\|g_{n}\right\|_{p^{\prime}}$ and $\left\|h_{n}\right\|_{p^{\prime}}$ are uniformly bounded.

Proof. If $T \in\left(L_{p}\right)$, then $\sup _{\|\rho\|_{p^{\prime}} \leqq 1}\left\|\int_{0}^{2 \pi} g(x) U_{n}(x ; t) d x\right\|_{p^{\prime}} \leqq K$. Also, 


$$
U_{n}(x ; t)=\sum_{k=0}^{n}\left(\frac{n+1-k}{n+1}\right)\left(g_{k}(t) \cos k x+h_{k}(t) \sin k x\right) .
$$

Let $g(x)=\cos j x, j \leqq n$. Then

$$
\int_{0}^{2 \pi} g(x) U_{n}(x ; t) d x=\pi g_{j}(t)\left(\frac{n+1-j}{n+1}\right) \quad \text { for all } n \geqq j .
$$

Thus,

$$
\pi\left(\frac{n+1-j}{n+1}\right)\left\|g_{j}(t)\right\|_{p^{\prime}} \leqq K, \text { or }\left\|g_{j}(t)\right\|_{p^{\prime}} \leqq \frac{K}{\pi}\left(\frac{n+1}{n+1-j}\right)
$$

for all $n \geqq j$.

For each $j$, we can choose $n$ so large that $((n+1) /(n+1-j)) \leqq 2$. Hence, $\left\|g_{j}(t)\right\|_{p^{\prime}} \leqq 2 K / \pi$. Similarly, $\left\|h_{k}(t)\right\|_{p^{\prime}} \leqq 2 K / \pi$.

Theorem 5. Let $T^{*}$ be the transpose of $T$. If $T \in\left(L_{p}\right), 1<p<\infty$, then $T^{*} \in\left(L_{p^{\prime}}\right)$.

Proof. Let $g \sim\left(c_{k}, d_{k}\right), g \in L_{p}^{\prime}$. Then

$$
T^{*}(g) \sim \sum_{j=0}^{\infty} \sum_{k=0}^{\infty} a_{k j}\left(c_{k} \cos j t+d_{k} \sin j t\right) .
$$

Consider

$$
\begin{aligned}
F_{n}(g ; t) & =\frac{1}{\pi} \int_{0}^{2 \pi} g(x) U_{n}(x ; t) d x \\
& \sim \sum_{k=0}^{n}\left(\frac{n+1-k}{n+1}\right) \sum_{j=0}^{\infty} a_{k j}\left(c_{k} \cos j t+d_{k} \sin j t\right)
\end{aligned}
$$

Since $T \in\left(L_{p}\right),\left\|F_{n}(g ; t)\right\|_{p^{\prime}} \leqq K$ for all $n$, by Theorem 2 . Let

$$
\sigma_{m}\left(F_{n}\right)=\sum_{j=0}^{m}\left(\frac{m+1-j}{m+1}\right) \sum_{k=0}^{n}\left(\frac{n+1-k}{n+1}\right) a_{k j}\left(c_{k} \cos j t+d_{k} \sin j t\right) .
$$

Then $\left\|\sigma_{m}\left(F_{n}\right)\right\|_{p^{\prime}} \leqq K$ for all $n$. By Fatou's lemma,

$$
\int_{0}^{2 \pi} \lim _{n \rightarrow \infty}\left|\sigma_{m}\left(F_{n}\right)\right|^{p^{\prime}} d t \leqq \lim \inf _{n \rightarrow \infty} \int_{0}^{2 \pi}\left|\sigma_{m}\left(F_{n}\right)\right|^{p^{\prime}} d t,
$$

or $\left\|\sigma_{m}\left(T^{*} g\right)\right\|_{p^{\prime}} \leqq K$, which is sufficient for $T^{*} \in\left(L_{p^{\prime}}\right)$.

Corollary. If $T \in\left(L_{p}\right), 1<p<\infty$, then there is a uniform bound to the $p^{\prime}$ th norms of the rows and the pth norms of the columns. 
Proof. This follows from Theorem 4.

TheOREM 6. Let $T \in\left(L_{p}\right)$ and $\left(L_{q}\right)$. Then $T \in\left(L_{r}\right)$ for any $r$ between $p$ and $q$.

Proof. By Theorem 1, T may be considered to be a linear bounded transformation from $L_{p}$ to $L_{p}$ and $L_{q}$ to $L_{q}$. Then, by the well known interpolation theorem of M. Riesz [7, p. 198], $T \in\left(L_{r}\right)$ for any $r$ between $p$ and $q$.

THEOREM 7. Every bounded linear transformation $T$ on $L_{p}$ to $L_{p}$ can be represented uniquely by a matrix transformation.

PRoof. In complex form, let $f \sim\left(c_{k}\right) \in L_{p}$. Let $s_{m}=\sum_{j=-m}^{m} c_{j} e^{i j x}$. Since $T(f) \in L_{p}$, let $T(f)=F \sim\left(d_{k}\right)$. Since $e^{i j x} \in L_{p}$ for each $j$, consider the Fourier expansion, $T\left(e^{i j x}\right) \sim \sum_{k--\infty}^{\infty} a_{k j} e^{i k x}$, where $a_{k j}$ $=(1 / 2 \pi) \int_{0}^{2 \pi}\left[T\left(e^{i j x}\right)\right] e^{-i k x} d x$. Then $T\left(s_{m}\right) \sim \sum_{j=-m}^{m} c_{j} \sum_{k=-\infty}^{\infty} a_{k j} e^{i k x}$, and if $c_{k}\left[T\left(s_{m}\right)\right]$ is the $k$ th coefficient in the Fourier expansion of $T\left(s_{m}\right)$, then

$$
c_{k}\left[T\left(s_{m}\right)\right]=\sum_{j=-m}^{m} a_{k j} c_{j} .
$$

Since $\left\|s_{m}-f\right\|_{p}=o(1)$, the continuity of $T$ implies that $\left\|T\left(s_{m}\right)-F\right\|_{p}$ $=o(1)$, from which it follows that $\lim _{m \rightarrow \infty} c_{k}\left[T\left(s_{m}\right)\right]=d_{k}$. That is, $d_{k}=\sum_{j=-\infty}^{\infty} a_{k j} c_{j}$.

The uniqueness of the representation follows from the uniqueness of the Fourier series expansion of a function in $L_{p}$.

It is also possible to give necessary and sufficient conditions for $T \in\left(L_{p}\right)$ in another form. The following is superficially similar to a theorem by Hille and Tamarkin [4] concerning $\left(L_{p}\right)$-effective matrices.

THEOREM 8. Necessary and sufficient conditions for $T$ to be in $\left(L_{p}\right)$, $1<p<\infty$, are:

(i) $T \in\left(L_{p}\right)$ on every subset $L_{p}^{0}$ which is dense in $L_{p}$, and

(ii) the set of transformations $\left\{T_{n}\right\}$ defined by $T_{n}(f)=\sigma_{n}^{T}(f ; x)$ form a uniformly bounded sequence of transformations on $L_{p}$ into itself. For sufficiency, condition (i) may be replaced by

(i') $T \in\left(L_{p}\right)$ on a single subset $L_{p}^{0}$ which is dense in $L_{p}$.

Proof. Necessity. The necessity of (i) is obvious. The necessity of (ii) is an immediate consequence of the proof of Theorem 1.

Sufficiency. Assume that (i') and (ii) are satisfied. Then an arbitrary function $f$ in $L_{p}$ can be approximated in $L_{p}$ as closely as we wish by a function of $L_{p}^{0}$. That is, if we are given an $f$ in $L_{p}$ and an arbitrarily 
small $\epsilon>0$, then we can find a function $g_{\epsilon}$ in $L_{p}^{0}$ such that $\left\|f-g_{\epsilon}\right\|_{p}$ $\leqq \epsilon / 3 K$, where $K$ is an upper bound to the moduli of the operations defined by $\sigma_{n}^{T}(f ; x)$. Since $g_{\epsilon}$ is in $L_{p}$, there exists an $N>0$ such that for $n, m \geqq N,\left\|\sigma_{n}^{T}\left(g_{\epsilon} ; x\right)-\sigma_{m}^{T}\left(g_{\epsilon} ; x\right)\right\|_{p} \leqq \epsilon / 3$. We may write $\| \sigma_{n}^{T}(f ; x)$ $-\sigma_{m}^{T}(f ; x)\left\|_{p} \leqq\right\| \sigma_{n}^{T}\left(f-g_{\epsilon}\right)\left\|_{p}+\right\| \sigma_{n}^{T}\left(g_{\epsilon}\right)-\sigma_{m}^{T}\left(g_{\epsilon}\right)\left\|_{p}+\right\| \sigma_{m}^{T}\left(g_{\epsilon}-f\right) \|_{p}$. Then, for $n, m \geqq N,\left\|\sigma_{n}^{T}(f ; x)-\sigma_{m}^{T}(f ; x)\right\|_{p} \leqq K \epsilon / 3 K+\epsilon / 3+K \epsilon / 3 K=\epsilon$. Since the space $L_{p}$ is complete, there exists an $F$ in $L_{p}$ such that $\| \sigma_{n}^{T}(f ; x)$ $-F(x) \|_{p}$ converges to zero. This permits us to say that $T(f)=F$ in $L_{p}$, or $T \in\left(L_{p}\right)$.

So far, the end cases, $T \in(L)$ and $T \in(B)$, have defied our efforts. However, if we retreat to a more restricted class of matrices, those for which $\sum_{j=0}^{\infty}\left|a_{k j}\right|<M$ for all $k$, certain further results may be obtained. First of all, the integral representation of Lemma 1 is valid for the entire range, $1 \leqq p \leqq \infty$. With this representation it is not difficult to demonstrate the following theorem [6].

ThEOREM 9. If $\sum_{j=0}^{\infty}\left|a_{k j}\right|<M$ for all $k$, a necessary and sufficient condition for $T \in(B)$ is that $\int_{0}^{2 \pi}\left|L_{n}(x ; t)\right| d t$ be bounded uniformly in $n$ and $x$.

For the other end point, we may summarize the partial results obtained in the following [6]:

THEOREM 10. If $\sum_{j=0}^{\infty}\left|a_{k j}\right|<M$ for all $k$, a necessary condition for $T \in(L)$ is $\int_{0}^{2 \pi}\left|L_{n}(x ; t)\right| d x \leqq K$ for all $n$ and $t$. Further, sufficient conditions for $T \in(L)$ are given by either of the following:

(i) $\int_{0}^{2 \pi}\left|L_{m}(x ; t)-L_{n}(x ; t)\right| d x<\epsilon$ for large $m, n$, independent of $t$;

(ii) (a) $\int_{0}^{2 \pi}\left|L_{n}(x ; t)\right| d t \leqq K_{1}$ for all $n$ and $x$, and

(b) $\int_{0}^{2 \pi}\left|L_{n}(x ; t)\right| d x \leqq K_{2}$ for all $n$ and $t$.

It may also be observed that if $T$ and $T^{*}$ are subjected to the condition of row boundedness used above, then $T^{*} \in(L)$ implies $T \in(B)$ [6].

The proofs of Theorems 9 and 10 and of the last remark will be omitted since the results are so markedly weaker than those for the open interval.

The author wishes to express his appreciation to Professor Paul Civin, whose help and suggestions were invaluable in the preparation of this paper.

\section{BIBLIOGRAPHY}

1. Richard Bellman, $A$ note on a theorem of Hardy on Fourier constants, Bull Amer. Math. Soc. vol. 50 (1944) pp. 741-744.

2. Michael Fekete, Über Faktorenfolgen, welche die "Klasse" einer Fourierschen Reihe unverandert lassen, Acta Litt. ac Sci., Szeged vol. 1 (1923) pp. 148-166. 
3. G. H. Hardy, Notes on some points in the integral calculus, Messenger of Mathematics vol. 58 (1929) pp. 50-52.

4. Einar Hille and J. D. Tamarkin, On summability of Fourier series. II, Ann. of Math. (2) vol. 34 (1933) pp. 329-348.

5. S. Verblunsky, On some classes of Fourier series, Proc. London Math. Soc. vol. 33 (1932) pp. 287-327.

6. F. H. Young, A matrix transformation of Fourier coefficients, Thesis, University of Oregon, 1950.

7. Antoni Zygmund, Trigonometrical series, Warsaw-Lwow, 1935, 331 pp.

UNIVERSITY OF OREGON

\section{A REMARK ON LINEAR ELLIPTIC DIFFERENTIAL EQUATIONS OF SECOND ORDER}

\section{EBERHARD HOPF}

Consider a linear partial differential expression

$$
L(u)=\sum_{i, k} a_{i k}(x) \frac{\partial u}{\partial x_{i} \partial x_{k}}+\sum_{i} b_{i}(x) \frac{\partial u}{\partial x_{i}}
$$

with no term $c(x) u$. The coefficients $a_{i k}$ and $b_{i}$ are suppose to be continuous in an open connected set $R$ of $x$-space, $x=\left(x_{1}, \cdots, x_{n}\right)$. Let $x^{0}$ denote a point on the boundary of $R$ which has the property that $R$ contains the interior of a hypersphere $\left|x-x^{*}\right|<r_{0}$ with $x^{0}$ on its boundary. Suppose that the coefficients are continuous at $x=x^{0}$ also. Let, finally, $L$ be elliptic in $R+x^{0}$ such that the quadratic form

$$
\sum a_{i k}(x) \lambda_{i} \lambda_{k}
$$

is positive definite in each point of $R+x^{0}$.

This note contains a simple proof of the following:

Theorem. Suppose that $u=u(x)$ is of class $C^{\prime \prime}$ in $R$ and that $u \geqq 0$, $L(u) \leqq 0$ in $R$. If the limit value of $u$ at $x=x^{0}$ is zero, then either the normal derivative $d u / d n$ at $x=x^{0}$, understood as the limit inferior of $\Delta u / \Delta n$, is $>0$ or $u \equiv 0$ in $R$.

Special cases of the theorem have been known for a long time. It contains, in particular, the fact that Green's function of $L$ has a positive normal derivative along the boundary if the boundary is sufficiently smooth.

To prove the theorem we note first that $u \geqq 0$ in $R$ and $u\left(x^{0}\right)=0$ trivially implies $d u / d n \geqq 0$. The hypotheses that $u \geqq 0$ in $R$ and $L(u)$ $\leqq 0$ in $R$ imply that either $u>0$ in $R$ or $u \equiv 0$ in $R$. This follows from

Received by the editors February 18, 1952. 